\title{
Framework for The Development of a Low-Cost Radio Frequency Identification (RFID) Based-System for Supporting Vaccine Immunization and Uptake in a Developing Country
}

\author{
${ }^{1}$ Longe, O.B, ${ }^{2}$ Onawola, H.J, ${ }^{3}$ Okolo, O., ${ }^{4}$ Adegbenro, D.A., ${ }^{5}$ Wada, F \& ${ }^{6}$ Chiemeke, S. \& ${ }^{7}$ Durosinmi, A.

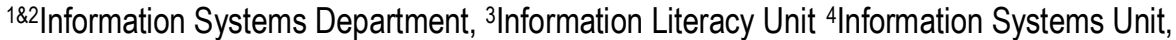 \\ American University of Nigeria, Yola, Adamawa State, Nigeria \\ ${ }^{5}$ Barney School of Business, University of Hartford, West Hartford, CT 06117 \\ ${ }^{6}$ CDepartment of Computer Science, University of Benin, Benin City, Nigeria \\ ${ }^{7}$ Information Technology Unit, Federal Medical Centre, Abeokuta, Nigeria \\ E-mails: ${ }^{13}$ olumide.longe@aun.edu.ng, 2hassan.onawola@aun.edu.ng, 30machi.okolo@aun.edu.ng; \\ 4dimeji.adegbenro@aun.edu.ng; 5Wada@hartford.edu; 6schiemeke@yahoo.com; ${ }^{7}$ cunlexie@hotmail.com
}

\begin{abstract}
Child Mortality in Africa has been attributed to the lack of access to major vaccinations as and at when due. Leveraging on existing cheap platform such as RFID Technology provides the overall availability of these vaccines to rural areas in sub-Saharan Africa can be improved. The goal is to create a practical system for improving vaccine uptake in pregnant women in sub-Saharan Africa, with Nigeria as a case study, and in all reducing child mortality. We employ mixed method such as action research and design science in this study with a view to involving the target population in the design of acceptable solution for the problem of vaccination uptake and administration.
\end{abstract}

Keywords- RFID, Africa, Resource poor environment, Vaccine, Vaccination, Uptake and Distribution.

Journal Reference Format:

Longe, O.B, Onawola, H.J, Okolo, O., Adegbenro, D.A., Wada, F \& Chiemeke, S. \& Durosinmi, A. (2020): Framework for the Development of a Low-Cost Radio Frequency Identification (RFID) Based System For Supporting Vaccine Immunization and Uptake in a Developing Country. Behavioural Informatics, Digital Humanities \& Development Journal Vol 6. No. 1. Pp 13-20 Available online at https://www.isteams.net/behavioralinformaticsjournal

\section{INTRODUCTION}

Child mortality rates are falling globally as a result of advances in vaccine production, but more progress is still expected. The 2019 UNICEF data showed that there has been a gradual decrease in the rate of child mortality between 1990s to date. The annual rate of reduction in mortality among the children that under-five has increased from 2.0 per cent in 1990 to 3.8 percent in 2018 (UNICEF, 2019). Many countries have shown considerable progress in tackling child mortality, almost one-third of the 49 least developed countries such as western Asia, North Africa and the Caribbean have managed to reduce their under-five mortality rate by 40 per cent or more over the past 20 years (UNICEF, 2019). By contrast, little progress has been made by countries, especially in Nigeria and other sub-Saharan Africa countries. Data from the Nigeria Demographic Health Survey (NDHS, 2018), showed that the under-five mortality rate in Nigeria is 132 per 1,000 live-births, the painful implication of this is that 1 in 8 Nigerian children never reach the age of 5 (UNICEF, 2019). World health organization (WHO, 2020) report on immunization revealed that most death among infants in developing countries and in Nigeria are vaccine preventable, these diseases are Diphtheria, Haemophilus, influenza, serotype b infection, hepatitis B, measles, meningitis, mumps, pertussis, poliomyelitis, rubella, tetanus, tuberculosis, and yellow fever. Nigeria has a precarious problem of infant mortality complicated by vaccinating the world's third largest birth rate within a culturally and socioeconomically diverse demography (McGavin et al., 2018). 
To this end, it is imperative to decipher a technologically viable alternative that can serve as an antidote to this needless and unwarranted death of infants in Nigeria. Based on the foregoing, this research seeks to propose the use of a low-cost mobile/ RFID based system using location aware request response model to ensure compliance to vaccination schedules and adequate coverage of mapped-out geographical location for effective and efficient uptake and distribution of vaccines for infants. This research would be carried out from a sampled rural population in the six geo-political zones in Nigeria in a bid to properly collect real-life data and show how this technology will help reduce infant and maternal mortality rates in Nigeria.

\subsection{RFID In Perspective}

Radio frequency identification (RFID) is a type of technology that enables electronic identification. It is widely deployed in many applications in recent times. RFID technology allows personal information to be saved using a low-cost chip attached to the patient or any device that relates to the patient (Longe, 2014). This innovative technology has tremendous potential to improve patient healthcare quality by improving patient identification and ambiguity that may arise during patient-physician and physician-physician interactions (Hu et al., 2015). Radio Frequency Identification (RFID) is a fast evolving technology that utilizes radio waves for data collection and transfer. Historically, RFID technology has strong application in supply chain management to track goods in warehouses and in transit to the desired location, but today it has application in multiple domains. RFID technology has many attractive benefits such as cost saving measures and increase in efficiency in many domains (Gulcharan et al, 2013).

RFID technology is also explored in the healthcare services because of its ability to capture data automatically without human intervention (Paaske et al., 2017). One of the most intriguing facts about RFID is that it is not a new technology. It has been around since 1945, it was originally used as a covert listening device, and not an identification tag, but it is considered to be a predecessor of RFID technology because even though it was passive, it became energized and activated by waves from an outside source. Another early work explaining RFID is the landmark paper by Henry Stockman, titled "communicating by means of reflected power", but the first commercial application involving RFID was in the 70's and 80's and they were concerned with asset identification in single location (Longe, et al. 2014).

The reason why the RFID technology will be a great benefit for the design of the artefact intended in this study is mostly due to its easy integration and structure, ability to read/write information remotely. This technology can help a group of $10-50$ persons made up of doctors, nurses, etc., have a reach of over 500 different persons. For this research, a case study will be carried out in one of the six geo-political zones of Nigeria in a bid to collect data and show how this technology will help reduce infant and maternal mortality rates in Nigeria.

\subsection{Problem Statement}

Accurate and routine vaccination is a perennial problem in Nigeria, the study conducted by McGavin et al. (2018) on the nature of immunization coverage among 5759 children within one year of age revealed that $25.5 \%$ of the children were fully vaccinated, $47.9 \%$ were under or irregularly vaccinated, and $26.6 \%$ had not received any vaccinations. This problem is exacerbated by myriad challenges of culture, illiteracy, unavailability of health care centers in rural areas, and poverty. Although research has shown that non-compliance with vaccination in most countries is caused by financial reasons (Harris Interactive, 2001), however, in resource-poor environments such as Nigeria, a preliminary interrogation of nursing mothers on non-compliance to vaccination regimen for infants revealed that lack of information, monitoring, lack of knowledge of medications, confusion with medication and forgetfulness are major factors responsible for non-compliance. Oku et al., (2017) revealed myriads of problem affecting vaccination communication and awareness in Nigeria, these included funding constraints, poor attitude of health workers and vaccination teams, inadequate infrastructure and equipment and weak political will, and health human resource factors. In an attempt to solve these problems, they proffered political-will and organized communication committee to effect workable vaccination communication. 
While these factors are admissible, their study failed to consider the use of emerging technology to effect inadequacy of communication problem in vaccination. This study proposed the use of technology to ensure compliance and fill up the research gap in vaccination communication by innovating a design of a low-cost RFID based system that can help improve vaccine uptake.

\subsection{Aim and objectives of the study}

The aim of this research is to solve the problem the infants' mortality and maternal health care with the use of lowcost mobile/RFID technology to administer vaccine and monitor compliance. The specific objectives are:

i. To investigate the level of vaccination compliance and schedule visit of infant and maternal patients in each six geo-political zones in Nigeria

ii. Develop a framework based on compliance and non-compliance based on the data collected

iii. Design a prototype device that can serve as prompt for infant and maternal patients for vaccine and schedule visit compliance.

\section{REVIEW OF RELATED LITERATURE}

There are prolific writings and studies on childhood mortality in Nigeria. This is predicated on the urgency of the problem and the need to provide workable solutions. Yaya et al. (2017) stressed on the menace of infant mortality to public health system amongst families in Nigeria. While echoing the worrisome situation, their study linked the high rate of infants' mortality to low-level of education, living standards and parents age. Similarly, McGavin et al. (2018) study on the nature of immunization coverage among 5759 children within one year of age revealed that $25.5 \%$ of the children were fully vaccinated, $47.9 \%$ were under or irregularly vaccinated, and $26.6 \%$ had not received any vaccinations.

Atkinson et al., (2016) conducted a survey to measure if the use of mobile app could lead to change in attitudes, and beliefs or behavior of participant to comply with vaccination time-table and reduce vaccine preventable deaths. The study revealed that the use of mobile app was a well-received technological solution, but there was a divergent view on the accessibility and application of the technology. Similarly, studies such as (Balakrishnan et al., 2016; Gatuha, G., and Jiang, T. 2015) proposed prototypes using mobile technologies and an open source data collection frameworks to test the feasibility of improving the vaccination data collection in Afghanistan and Kenya. The proposed proto-types offers ways of collecting vaccination data through mobile phones and visualizes the collected data in a web application; the system also sends reminder short messages service (SMS) to remind parents on the date of the next vaccination. Early evaluation demonstrates the benefits of such a system in supporting and improving vaccination of children.

While these earlier proposal based on mobile health (mobile health) tried to address vaccination compliance problem, our proposed prototype improve the existing technologies by including portable artefacts given to parents as well added functionalities such as: communicating to parents in their local languages, alarm, creation of central database that can be used to further investigate other vaccine preventable disease. Our study would also investigate the behavioral and other underlying issues affecting immunization in the six geo-political regions of Nigeria with a view to developing a local framework that can uncover local patterns and problems 


\section{PROPOSED METHODOLOGY/SYSTEMS DESIGN}

This study combines both action research and design science methods. The action research paradigm involve the clients or the target population in finding solutions to the problem, the design science method would use the data collected in order to prototype and coming up with the artefacts intended to solve the problem of infant and maternal vaccine uptake. Quantitative data would be collected using questionnaire from a sampled population of the target community.

\subsection{System Design}

The design of this low-cost RFID artefact is intended to improve vaccine uptake in pregnant women and children. The proposed Mobile/RFID technology would be a small portable device given to mothers upon visit to health centers for mothers and children related care and vaccinations, the device would be designed in a multifunction mode such as:

a) An inbuilt periodic alarm

b) Ability to send periodic SMS/alerts to the infant mothers' through phone with the help of translator which translates in a three Nigerian languages.

c) A sensor Tags which senses the activities in (a) and (b)

d) Readers: As described above, the readers will be stationary, residing in the test location.

e) RFID Middleware: consists of an Electronic Patient Records System (EPRS), Database Management Systems and an SMS Server System.

f) Mobile Wireless Network with Voice capability.

This technology would help track all pregnant and infant mothers captured and registered in the database and help them to respond timely to ante-natal and post-natal care and other routine immunization after the first registration at a medical care center. In addition to providing a technological artefact that can enhance prompt access to uptake of vaccine, the research will also seek to educate parents, (especially mothers), using their own native language about the importance of immunization and vaccination, also provide basic medical and nutritional advise on food supplements that can be taken to combat malnutrition as is the case in Nigeria.

The framework for this research is the Self-Regulation Model (SRM) used for patient compliance administration. SRM is a common-sense framework best suited for processes by which patients become aware of a health challenge, navigate effective responses to counter the effect, formulate perceptions on how to deal with the disease and potential treatment, create action plans for addressing the diseases, and integrate continuous feedback on the desired action plan efficacy and the eventual threat-progression (Leventhal et al., 2016). The operation of this model would be managed and tested via the feedback loop from the patient. In this case the patient compliance with the vaccine time-table would be assessed and integrated with the designed artefact. Regimen-uptake is examined by the patients self-reports compared with the recommended treatment regimen. Non-compliance can be detected when there is a disparity between the recommended and actual treatment regimen.

Compliance is facilitated through social networks (Using the social construction theory; the use of reminders and alerts (Based on associative theory) and questionnaires based on self- perception theory. While these contributions are noteworthy, it is imperative to assert that in a resource poor environment, most patients do not possess the skill, experience, resources and motivation to use the compliance facilitators prescribed by SRM. We propose to develop a robust means of integrating low cost location detection system alongside biometric technology to enforce compliance, provide real-time alerts/signals that will tremendously improve uptake with infant vaccination and connect healthcare givers/vaccinators with target populations using a Location Aware Request Response mechanism (LARRM). 
The basis for using the LARRM Mechanism for the development of low-cost mobile platform is to leverage on existing mobile networks and explore ICT4D2.0 scientific imperatives by adding wireless/web functionality and digital/geographic mapping tools (Google maps) to legacy technologies such as radios and televisions in places that are out of the reach of mobile technologies. This will assist to provide timely information on the availability and location of infant vaccinations offering in a bid to improve participation, enhance coverage and facilitate adequate penetration of the exercise. To overcome the challenges of unimodal biometric systems, Multibiometrics regimen will be engaged for enrolment, verification and identification of infants. This will be stored in a database as a set of robust infant biometric data that can be used to validate infant patient information with the aim of identifying individual infants for vaccination uptake and promoting compliance with respect to vaccinepreventable diseases. Google Android technology and other smart phones with in-built GPS and accelerometers will be engaged to provide location information in environments where phones are available.

We expect that the project will be successful as it will assist in overcoming the specific factors identified as militating against effective uptake and compliance with infant vaccination regimen such as lack of information, the challenges of positively identifying individual infants for developing world vaccination programs and connecting vaccine availability with target population.

\section{EXPERIMENTAL PLAN AND TESTING}

The initial effort will be geared towards evaluating the success of vaccination in the research locations by tracking the number of children that have been vaccinated, their effectiveness cum efficiency with a view to connecting vaccine availability with target population. This will be followed by development of a biometric database to assist in verification and authentication of existing patients with future growth in mind.

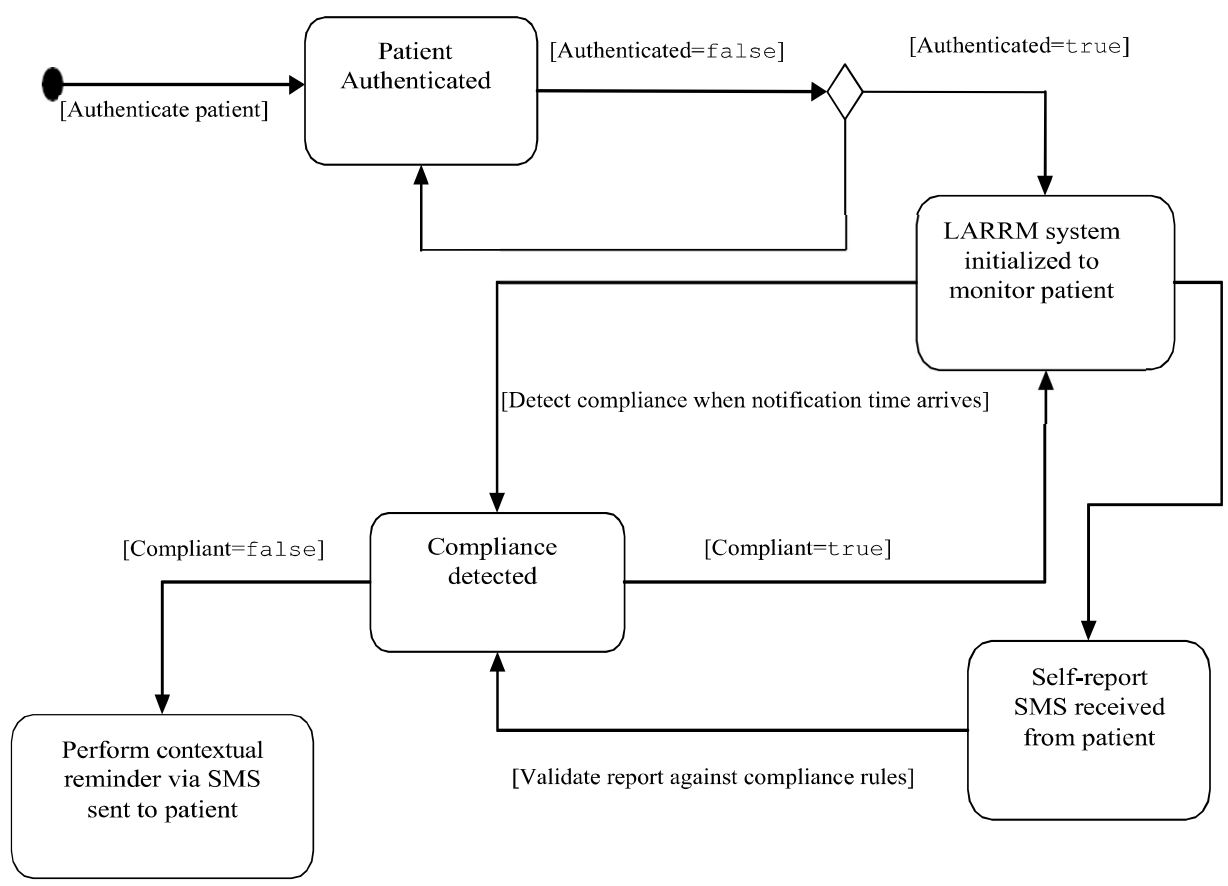

Figure 1: The LARRM Mechanism for Ensuring Uptake and Compliance of Vaccination in the Developing World 
In places where there are no cell phone access we shall employ radio jingles and leverage on existing telecommunication infrastructure in combination with Radio Frequency Identification Tags (RFID tags) placed in homes with infants whose biometrics have already been taken connected to antennas and radio sets/megaphones (powered by solar cells/solar panels ) to provide beeping information which eventually generates announcements in local languages to inform parents of imminent vaccinators visits, time and location. We will use the Social Learning Theory (SLT) as a basis for assessing incremental and transformative changes occasioned by the use of mobile and telecommunications technology on compliance intervention strategies for the distribution and uptake of infant vaccination

\subsection{Data Generation}

Comprehensive information on local population depicting the ratio between vaccinated and potential infants to be vaccinated of the chosen locality as well as basic patient demographics, treatments, diagnoses, laboratory, and other test data.

- Individual biometric identity data verifiable on the fly to determine the level of compliance, vaccine penetration, efficacy of the vaccine and a digital map of the case studies will be produced. If successful, the LARRM based mechanism would be developed as a mass produced low cost device for rapid and accurate dissemination of vaccination information and model for the effective measure for the uptake and distribution of infant vaccination in the developing world.

\subsection{The Pilot System}

How the pilot system will operate and function will be described in the "General System Description". Some key benefits of the system are:

a) Searching for patient records will be easy and more simplified.

b) The system will allow for easy modification of any data entered on the system, and that to be sentout.

c) The information can be easily accessed remotely.

d) The system will be conveniently cross-referenced, maintained and updated, and protected.

e) Most importantly, the system will help reduce infant mortality rates in the Country be making sure that the important vaccines are taken at the appropriate time during infant development.

\subsection{General System Architecture.}

\section{The Database}

The database system will be computed-based, php run or any other thing. It might even be in form of a software. The basic idea is that the database stores information of each patient (child \& patient) as regards personal information, location information, health history and any other relevant information. To overcome negligence on the part of the person operating the database, clinic cards would be used so that we can have access to information from two different sources, which will reduce errors.

\section{Computer Networks}

This comprises of the RFID software and hardware systems that will be located at the hospital or clinic premises. The sole purpose is to interface the information from the reader/interrogator to the database and also send and update information through the reader to the tag. The mode of communication is through Mobile Data Transfer through the GSM Network. The authentication protocol for reading information on the tag will basically be by File Transfer Protocol, as this will save cost of communication through the Mobile GSM Network. 


\section{Reader/Interrogator}

This will send the signal to the tag and read responses from the tag. They will generally transmit their information/observation to the computer network running the RFID middleware or software. The reader will also capture and process the tag data. An important aspect of the transmission of information between tag and reader is the frequency, which defines the data transfer rate. It should be noted that higher frequency means smaller antenna, smaller tag size, greater range but higher cost. This paer would recommend that the microwave $2.45 \mathrm{ghz}$ and $5.8 \mathrm{ghz}$ or higher should be used. Though it might be subject to poor performance around liquids and metals. The reader will be situated at strategic positions in the test area so that it can read for the tags in that specific geographical location. For example, reader R1 might serve for tags $\mathrm{T} 1, \mathrm{~T} 2, \mathrm{~T} 3, \mathrm{~T} 4, \mathrm{~T} 5$ respectively while reader $\mathrm{R} 2$ will serve for tags $\mathrm{T} 6, \mathrm{~T} 7, \mathrm{~T} 8, \mathrm{~T} 9, \mathrm{~T} 10$ and both make up the RFID cloud.

\section{Tags}

When a tag is interrogated, data from its memory is retrieved and transmitted. Tags can perform basic tasks of read/write from/to a memory location. There are two types: active and passive, but for the scope of our project, active tags should be used because they have more range, more complex information exchange and richer processing capabilities. The only problem with the active tags is the battery life.

Table 2: Tagging:

\begin{tabular}{|l|l|}
\hline Attribute & Characteristics \\
\hline Design & IC based (so as to store information) \\
\hline Type & $\begin{array}{l}\text { Active (requires battery to operate the IC and to communicate with the reader. It also } \\
\text { offers highest range } \\
\text { and accuracy. Though it is costlier than passive tags.) }\end{array}$ \\
\hline Memory & Read/write type \\
\hline
\end{tabular}

To fit this description and also for patient satisfaction, tags that come in form of Wristwatches should be employed as this removes the problems attached to privacy and general acceptance of the RFID technology.

\section{SMS SERVER.}

In addition, an SMS Server will be needed basically to send text messages to patients reminding them of appointment times to come for vaccination. The idea is that the SMS will remind the patient of the time for the vaccination, day, doctor, name of vaccine, e.t.c. Better still the Mobile SMS Network would have VoiceCapability so that the text message will be read out or a phone call in the native language of the people in that particular geographic location. To accomplish this we can either use the Mobile Med Alert System as described in "The Design of a Mobile-Based Alert System for Outpatient Adherence in Nigeria" or tweak its architecture to suit our purpose, but I must say that the architecture of the Mobile Med Alert System is good enough to give us what we need exactly.

\section{CONCLUDING REMARKS}

We have proposed and discussed a framework for the design of a low-cost radio frequency identificationbased system for supporting vaccine distribution and uptake in resource poor environments. Although research has shown that non-compliance in most advanced countries is caused by financial reasons (Harris Interactive, 2001), in resource-poor environments such as Nigeria, a casual interrogation of nursing mothers on non-compliance to vaccination regimen for infants revealed that lack of information, monitoring, lack of knowledge of medications, confusion with medication and forgetfulness are major factors responsible for noncompliance. 
The development and implementation of our proposed system is expected to use a location aware request response model to ensure compliance to vaccination schedules and adequate coverage of mapped-out geographical location for effective and efficient uptake and distribution of immunization vaccines for infants.

\section{REFERENCES}

1. Atkinson, K. M et al. (2016). Can mobile technologies improve on-time vaccination? A study piloting maternal use of ImmunizeCA, a Pan-Canadian immunization app. Human Vaccines \& Immunotherapeutics, 12(10), 2654-2661. https://doi.org/10.1080/21645515.2016.1194146

2. Balakrishnan, R., et al. (2016). Continuum of Care Services for Maternal and Child Health using mobile technology - a health system strengthening strategy in low and middle income countries. BMC Medical Informatics and Decision Making, 16(1), 84. https://doi.org/10.1186/s12911-016-0326-z

3. Elwy, A. R., Yeh, J., Worcester, J., \& Eisen, S. V. (2011): An Illness Perception Model of Primary Care Patients' Help Seeking for Depression. Qualitative Health Research, 21(11), 1495-1507. https://doi.org/10.1177/1049732311413781

4. Hu, L., Ong, D. M., Zhu, X., Liu, Q., \& Song, E. (2015): Enabling RFID technology for healthcare: application, architecture, and challenges. Telecommunication Systems, 58(3), 259-271. https://doi.org/10.1007/s11235-014-9871-x

5. Leventhal, H., Phillips, L. A., \& Burns, E. (2016): The Common-Sense Model of Self-Regulation (CSM): a dynamic framework for understanding illness self-management. Journal of Behavioral Medicine, 39(6), 935-946. https://doi.org/10.1007/s10865-016-9782-2

6. Longe, O.B et al. (2014): Towards the development of a Radio Frequency Identification (Rfid) based system for supporting vaccine distribution and uptake for Infants. International Conference on Science, Technology, Education, Arts, Management and Social Sciences iSTEAMS Research Nexus Conference, Afe Babalola University, Ado-Ekiti, Nigeria, May, 2014.

7. McGavin, Z. A., et al. (2018). Childhood full and under-vaccination in Nigeria. Vaccine, 36(48), 72947299. https://doi.org/10.1016/j.vaccine.2018.10.043

8. Paaske, S., Bauer, A., Moser, T., \& Seckman, C. (2017). The Benefits and Barriers to RFID Technology in Healthcare - ProQuest. On - Line Journal of Nursing Informatics, 21(2). https://search.proquest.com/openview/246b89e011c35482a3103c9de7c592fb/1?pqorigsite $=$ gscholar $\& \mathrm{cbl}=2034896$ 